\title{
Portal de Periódicos UFSC
}

\section{Ursula Blattmann}

\author{
Florianópolis, 6 de maio de 2008
}




\section{Uma história a ser contada...}

- Projeto: CIN - CED - 2007

- Apoio : UFSC PRCE, PRPG, PRP, CED, CIN, PGCIN, Encontros Bibli e BU/UFSC.

- Equipamento - servidor - NPD - out.2007, OJS 2.1

- Início das atividades: configurações, testes, equipamentos e políticas internas e externas

- Metas: preservação da memória institucional, disseminação e recuperação da informação e a visibilidade científica. 


\section{Cultura organizacional}

- Processo de produção de revistas

- Processo de publicação de artigos

- Processo de avaliação pelos pares

- Processo de indexação nacional e internacional

- Auditoria no processo de qualidade

- Impacto de periódicos científicos da UFSC 


\section{Qualidade X Quantidade}

- 53 Periódicos científicos, 42 com Qualis,

3 na SciElo, 32 no portal, 7 completas.

- Investir na formação e qualificação dos editores dos periódicos da UFSC

- Troca de experiências entre editores, bolsistas e equipes

- Visibilidade aos periódicos científicos 


\section{Alguns resultados}

Oficinas, cursos, disciplinas e palestras

Edições das Coleções Completas :

1. Encontros Bibli - $\mathbf{3 1}$ edições

2. Boletim de Pesquisa NELIC -8 edições

3. Esboços -17 edições

4. Ponto de Vista -6 edições

5. Revista Contemporânea de Contabilidade -7 edições

6. Revista Internacional Interdisciplinar INTERthesis - 8 edições

7. Texto Digital $-\mathbf{5}$ edições

Estatísticas

http://www.periodicos.ufsc.brlawstats/awstats.pl 


\section{Portaria n. 918/GR/2007 UFSC}

- De 06 de dezembro, retroativa a 01 de outubro de 2007.

- Designa os professores Rosângela Schwarz Rodrigues, Ursula Blattmann, Raimundo Nonato Macedo dos Santos, Araci Isaltina de Andrade Hillesheim e os servidores técnicos administrativos Kathia Regina Lemos Jucá e Maristela Helena Zimmer Bortolini.

- "Tratar das questões operacionais para a viabilização do Portal dos Periódicos da UFSC", relacionadas às atividades que já vinham sendo delineadas desde fevereiro de 2007. 


\section{Equipe do portal Periódicos UFSC}

Professores

- Rosângela Schwarz Rodrigues

- Ursula Blattmann

- Raimundo Nonato Macedo dos Santos

- Araci Isaltina de Andrade Hillesheim
Servidores técnicos administrativos

- Kathia Regina Lemos Jucá

- Maristela Helena Zimmer Bortolini

- Bolsistas PRCE

- Bolsistas de permanência

- Monitorias... 


\section{TENDÊNCIAS}

- Preservação da memória: concluir o processo de migração das revistas: configuração personalizada, metadados, formatação dos arquivos em PDF/A.

- Disseminação e Recuperação da informação: indexação (PKP, Google Scholar. Redalyc), diretórios e repositórios nacionais e internacionais (OASIS.BR, DOAJ, Scientific Commons), portais de acesso livre (Portal de Periódicos Capes)

- Visibilidade: PKP, Portal SEER, OASIS.BR, Portal Periódicos Capes, Acesso Livre, Google, E-ZEIT, DOAJ... 


\section{Ações para 2008}

- Colaboração com a comissão editorial da UFSC para definir os critérios de inclusão dos periódicos no portal, o design gráfico do portal e a política institucional para as publicações eletrônicas;

- Fortalecer a pesquisa associada às melhorias e atualização do programa SEER-OJS, juntamente com o IBICT;

- Manter em fluxo contínuo a capacitação e formação de recursos humanos para atuar nos processos de editoração científica, gestão de conteúdos digitais, para melhorar a visibilidade científica.

- Aprimorar os estudos sobre metadados para indexação internacional.

- Adoção e distribuição do Digital Object Identifier (DOI) pela UFSC. 


\section{http://redalyc.uaemex.mx/}

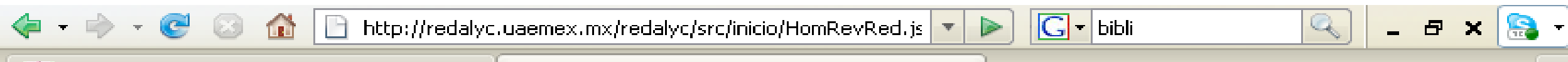

(1) Depto de Ciencia da Informaçao - UFSC $\square$ Redalyc - La hemeroteca científi...

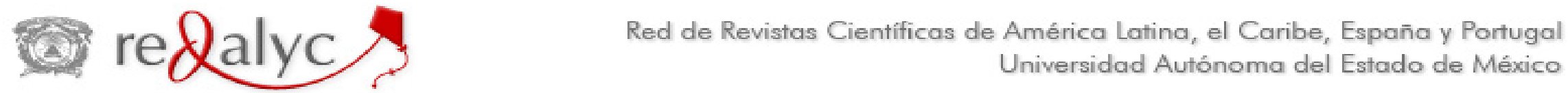

Encontros Eibli

Erasil

ISSN Electrónico:1518-2924

Universidade Federal de Santa Catarina

MNNO NULMERO

\begin{tabular}{|c|c|c|}
\hline 2007 & $\underline{024}$ & $\underline{023}$ \\
\hline 2006 & $\underline{022}$ & $\underline{021}$ \\
\hline 2005 & $\underline{020}$ & 019 \\
\hline 2004 & $\underline{099}$ & $\underline{018}$ \\
\hline 2003 & $\underline{016}$ & $\underline{015}$ \\
\hline 2002 & $\underline{014}$ & $\underline{013}$ \\
\hline 2001 & $\underline{012}$ & $\underline{011}$ \\
\hline 2000 & $\underline{010}$ & $\underline{009}$ \\
\hline 1999 & $\underline{008}$ & 007 \\
\hline 1998 & $\underline{006}$ & $\underline{005}$ \\
\hline 1997 & $\underline{004}$ & $\underline{003}$ \\
\hline 1996 & 002 & $\underline{001}$ \\
\hline
\end{tabular}

Sitios relacionados:

Encontros Bibli

iit

Portal desarrollado y mantenido por la Universidad Autónoma del Estado de México

\section{Estadísticas}

Artículos más consultados

Normas de colaboración

Características de la publicación

Indizaciones

Directorio

Contacto 


\section{http://www.doaj.org/}

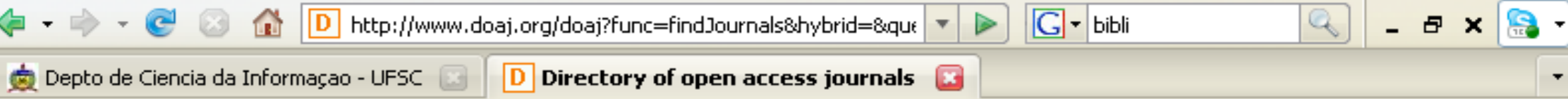

$0 \backsim \wedge \int \begin{aligned} & \text { DIRECTORY OF } \\ & \text { OPEN ACCESS } \\ & \text { JOURNALS }\end{aligned}$

Find Journals

\section{Find journals}

New titles

Find articles

Suggest a journal

bibli $\quad$ Find journals

found 1 journals matching your query

About

FAQ

News

Encontros Bibli: revista eletrônica de biblioteconomia e ciência da

informação [D0A.] Content

ISSN: 15182924

Sponsors

Subject: Library and Information Science

Membership

Publisher: Universidade Federal de Santa Catarina - Ciência da Informação Country: Brazil

Contact

Language: Portuguese, Spanish

Keywords: library science, information science, information management Start year: 1996

For journal owners

For authors

DOAJ - Directory of Open Access Journals, 2008, Lund University Libraries, Head office 


\section{http://rzblx1.uni-regensburg.de/ezeit/}

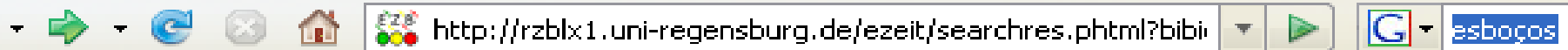

\section{Electronic Journals Library}

एक

Our Service I Preferences

\begin{tabular}{|l|}
\hline Journals \\
\hline - by sub ject \\
\hline - by title \\
\hline - Search \\
- quick Search \\
bibli \\
\hline search \\
\hline
\end{tabular}

pay per view

ppv offers by

publishers

Contact

Contact address

Sugqest a journal

1 hit(s)

Refine Search

Esbocos: Revista do Programa de Pós-Graduação em História da UFSC

Publisher:

P-ISSN(S):

ZDB-Number:

Subject(s):

Keyword(s):

Homepage(s):

fulltext online

since:

Type of

appearance:

Pricetype:
Universidade Federal de Santa Catarina

1414-722x

2423821-1

History

Regionalgeschichte Südamerika

http://w'w periodicos.ufsc.br/index.php/esbocos

Vol. 13, Iss. 15 (2006)

Fulltext, online and print

free of charge

Please respect the publisher's terms of use. 


\section{http://www.latindex.unam.mx/}

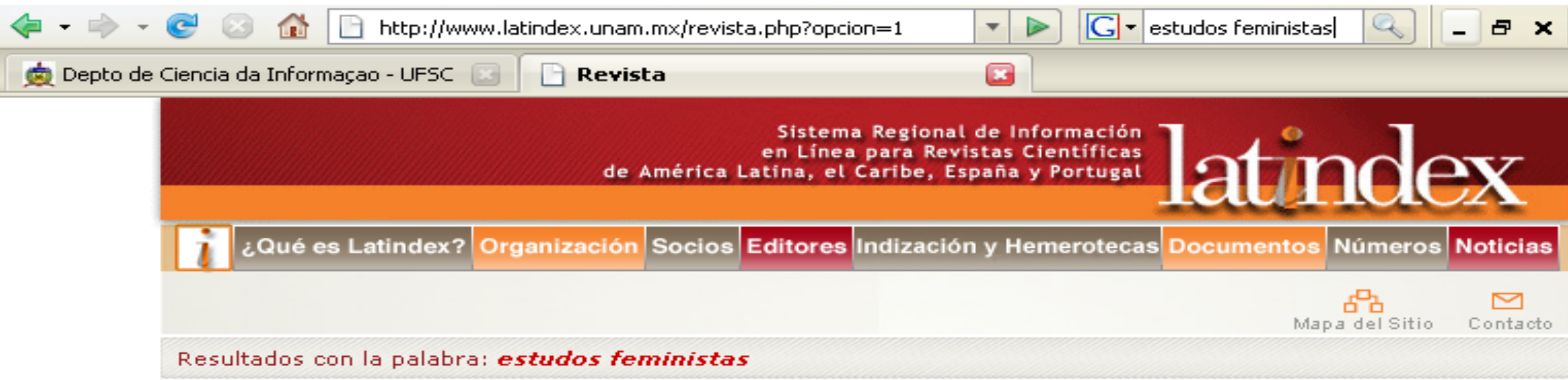

Revista estudos feministas (lmpresso) En Catálogo Editorial: Universidade Federal de Santa Catarina, Centro de Filosofia e Ciências

País: Brasil

Tema: Sociología;

ISSN: 0104-026X

มก๊̃o Inicio: 1992

Revista estudos feministas (online) En Catálogo * Revista electrónica

Editorial: Universidade Federal de Santa Catarina, Centro de Filosofia e Ciências

Humanas, Centro de Comunicação e Expressão

Pais: Brasil

Tema: Sociología;

ISSN: $1806-9584$

Aก๊o Inicio: 2001 


\section{http://pkp.sfu.ca/node/1468}

장 New OJS Journals from Brazil | Public Knowledge Project - Mozilla Firefox

Ärquivo Éditar Exibir ㅂistórico Favoritos Eerramentas Ajūda

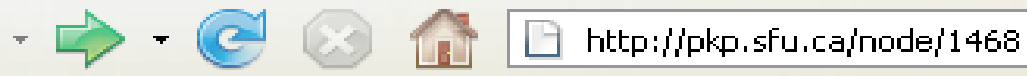

\section{PKP}

PUBLIC

KNOWLEDGE

PROJECT

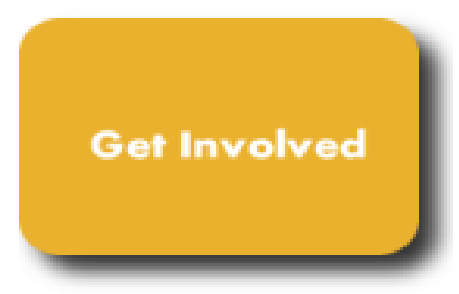

\section{Navigation}

- About PHP

- Prop Partners

- Software and Services

- Open Journal systems

- Open Conference systems

- Open Archives Harvester

- Lemon8-jo-rL

- Open Monograph PTess

- Theme Gallery
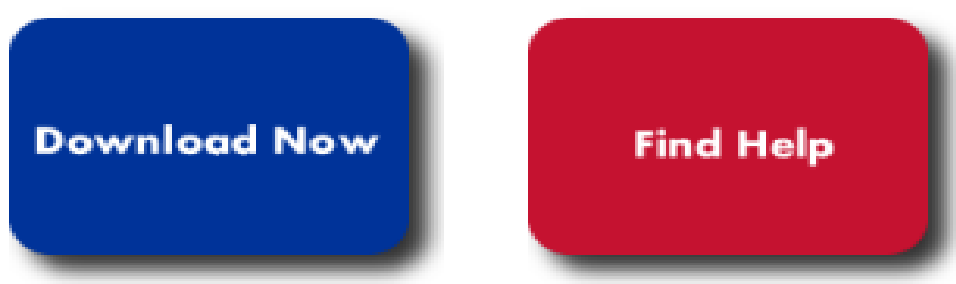

Home

\section{New OJS Journals from Brazil}

The Universidade Federal de Santa Catarina (UFSC) in Brazil has set up O.JS with an impressive list of $\mathbf{3 6}$ journals. The pace of work in Brazil is quite amazing. Nice work!

By kevin on 2008-04-04 07:03

| ojs | add new comment

\section{PKP Blog}

- Harry Potter and the Scholar's Fair Use May 2, 2008

- Video: Teaching for a World of Increasing Access to Knowledge April 30, 2008

- SPARC Europe and the Directory of Open Access 


\section{http://seer.ibict.br/}

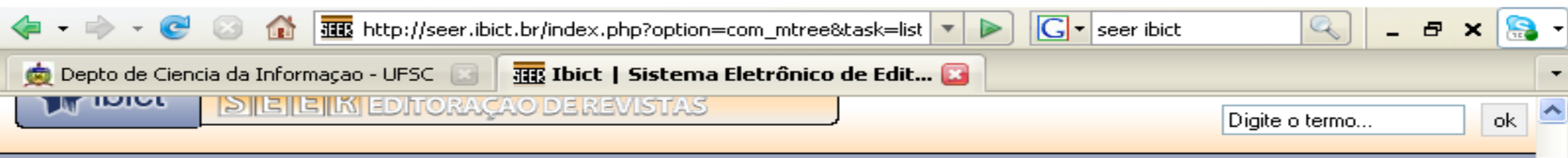

Início Revistas no SEER Instituiçä́o Universidade Federal de Santa Catarina - UFSC

SEGUNDA, 05 DEMAIO, 2008

\section{NAVEGAÇÃO}

\section{Início}

Notícias

Revistas no SEER

Treinamentos

Fale conosco

Documentos úteis

Downloads

\section{Equipe}

Perguntas frequientes

Fórum

Links relacionados

\section{AUTENTICAÇÃO}

Logir

Senha

$\square$ Memorizar

\section{REVISTAS NO SEER}

UNIVERSIDADE FEDERAL DE SAHTA CATARINA - UFSC

\section{Registros}

Total de registros: 3

Incluir registro aqui

Encontros Bibli: Revista Eletrônica de

Biblioteconomia e Ciência da Informação

Acesse a revista clicando aquil

Encontros Bibli: revista eletrônica de Biblioteconomia e Ciência da Informaçẫo tem como missẫo difundir o conhecimento novo e inovador em Biblioteconomia e Ciência da Informaçẫo, abrangendo interesses técni ...

\section{Cidade Florianópolis JWF SC Grande Area Não Informado}

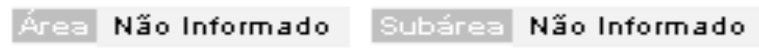

Äreas Correletes/Especialidedes Não Informado

ISSN Impresso Não Informado ISSN Eletrônico 1518-2924

Digital Dbject Identifier-DOI Não Informado

\section{Esboços}

Acesse a revista clicando aquil

A revista Esboços, vinculada ao Programa de Pós-Graduaçẫo em
PESQUISAR REVISTAS

Digite o termo..

Enviar

Pesquisa Avançada

\section{AJUDA}

A. ferramenta executa pesquisa exatamente pela(s) palavra(s) digitada(s).

Utilize o caracter asterisco ( $\left.{ }^{*}\right)$ como curinga para ampliar o leque de possiveis resultados

Ex.: Caderno* pesquisará Caderno, Cadernos, etc. 


\section{http://scholar.google.com.br/}

\section{Q. G http:i/scholar.google.com.brischolar?hl=pt-BR\&dr=\&safe=i}

\section{G. Google}

\section{Google}

\section{Pesquisar}

- Pesquisar na veb
Pesquisa aưançada do Google Acadêmico

Preferências do Google Acadêmico

Áuda do Google Acadêmico

\section{Acadêmico Todos os artigos - Artigos recentes Resultados 1 - 10 de aproximadamente 66 para Interthesis com Safesearch}

[cıтaçŭo] Da teologia política à teologia econômica-Entrevista com Giorgio Agamben

$G$ SACCO - Interthesis

Citado por 2 - Artigos relacionados - Pesquisa na web

Página inicial> Vol. 2, No 1 (2005) > Krischke

P.J Krischke - Revista Internacional Interdisciplinar INTERthesis, 2005 - periodicos.ufsc.br Revista Internacional Interdisciplinar INTERthesis, Vol. 2, No 1 (2005)....

Em cache - Pesquisa na web

\section{Página inicial> Vol. 1, No 1 (2004)> Raffaelli}

R Raffaelli - Revista Internacional Interdisciplinar INTERthesis, 2004 - periodicos.ufsc.br

Revista Internacional Interdisciplinar INTERthesis, Vol. 1, No 1 (2004)....

Em cache - Pesquisa na web

\section{Página inicial> Vol. 1, No 2 (2004)> Raffaelli}

R Raffaelli - Revista Internacional Interdisciplinar INTERthesis, 2004 - periodicos.ufsc. br

Revista Internacional Interdisciplinar INTERthesis, Vol. 1, No 2 (2004)....

Em cache - Pesquisa na web

[Pof] Sequndo Morais (1955, p. 32) assinala"( ..) a presença de concreçôes de bauxita no Rio Cricon". - todas as 2 Versổes»

E de Citaçốes - interthesis.cfh.ufsc.br

... superior. Contato com a INTERthesis 1) Preferencialmente, via correio

eletrônico: interthesis @cfh.ufsc.br 2) Via correio: Prof. Dr ...

Ver em HTWL - Pesquisa na web 


\section{Agradecimentos}

- Colegas do CIN e PGCIN. Rosângela, Francisco, Gleisy, Araci, Raimundo, Angel, Miriam. Andrenizia e Káthia.

- Apoio das instâncias acadêmicas: PRCE, PRPG, PRP, CED, PGCIN, BU/UFSC e NPD.

- Aos editores, bolsistas, monitores.

- PRCE: Eunice, Rose, Maristela e Vicenzo.

- Aos amigos Suely Soares (UNESP), Angélica Miranda (FURG), Diego Abadan e Marli Machado (Revista ACB). 


\section{... a história continua}

- Digital Preservation Coalition (DPC), Portable Document Format (PDF) is one of the best file formats to preserve electronic documents and ensure their survival for the future. This announcement will allow information officers to follow a standardised approach for preserving electronic documents.

- Betsy Fanning, author of the report and director of standards at AlIM, comments, "A standardised approach to preserving electronic documents would be a welcome development for organisations. Without this we could be walking blindly into a digital black hole."

- www.dpconline.org/graphics/reports/index.html\#twr0802 